\title{
One-Dimensional Wave Animation Using Mathematica
}

\author{
J. T. CHEN, ${ }^{1,2}$ K. S. CHOU, ${ }^{1}$ S. K. KAO ${ }^{1}$ \\ ${ }^{1}$ Department of Harbor and River Engineering, National Taiwan Ocean University, Keelung, Taiwan \\ ${ }^{2}$ Department of Mechanical and Mechatronic Engineering, National Taiwan Ocean University, Keelung, Taiwan
}

Received 16 August 2007; accepted 23 November 2007

\begin{abstract}
The work presents how one-dimensional wave phenomenon is animated. Several methods including the D'Alembert solution, the diamond rule, the Laplace transform and the convolution integral, are employed in the Mathematica animation. All the analytical derivations were carried out by using the symbolic software. Several examples, including an infinite string with a spring, mass and damper as well as a semi-infinite string, two-media string, string and beam subject to support motions, were demonstrated to show the validity of the present formulation. Parameter study of impedance ratio and mass, spring, and dashpot was also examined to see the transmission and reflection coefficient. (c) 2009 Wiley Periodicals, Inc. Comput Appl Eng Educ; Published online in Wiley InterScience (www.interscience.wiley.com); DOI 10.1002/cae.20224
\end{abstract}

Keywords: wave equation; diamond rule; series solution; animation

\section{INTRODUCTION}

One-dimensional wave problems have attracted much attention since early last century and have been studied both theoretically and experimentally due to its simplicity in teaching the engineering mathematics. The wave problem can be modeled as a second-order partial differential problem, which can be solved by engineering mathematics. Application of the symbolic software computation to engineering analysis was already a success by 1990 [1]. The computational tools of symbolic algebra such

Correspondence to J. T. Chen (jtchen@ ntou.edu.tw). (C) 2009 Wiley Periodicals Inc. as Mathematica, Reduce, Macsyma, and Maple were introduced in the course to solve problems as numerical experiments for an infinite string with spring, mass and damper as well as semi-infinite string and two-media strings, etc. Many researchers have solved wave problem in different branches of engineering. Wave reflection and transmission in thin tubes with initial deformation have been studied for the pulse propagation of blood in an artery [2]. A string subjected to support motion was solved by using the dual boundary integral equation (DBIE) [3]. The dynamic response of the Rayleigh-damped Bernoulli-Euler beam subjected to support motion was also solved by using the quasi-static decomposition method, Cesàro sum technique, and Stokes' 
transformation [4]. The hyperbolic type partial differential equation (PDE) for water-hammer equation and open-channel transient flow equations can be transformed to a characteristic form [5]. The reflection and transmission of harmonic elastic waves in a bent bar was investigated in Ref. [6]. Besides, the one-dimensional wave problem in non-uniform waveguides were solved and presented [7].

We may have interest to know how to capture the physical behavior of the one-dimensional wave problem theoretically, numerically, and experimentally. Although it is important to derive the wave solution, it is more important to educate students and engineers to understand the physical significance of wave phenomenon from the viewpoint of engineering education. Symbolic computation software has been employed to derive the solution of engineering problem for several years. Kaw and Ho [8] used three methods, the finite difference method, the finite element method, and the boundary element method to demonstrate the Lamé problem by using Maple. Symbolic software may be convenient for students, engineers, and researchers to work with computer than with a pencil. But, there are only a few articles to discuss how to combine education with software of symbolic computation.

In this article, we will solve one-dimensional wave problems using the D'Alembert's solution, the diamond rule, the image method and the series solution. In the textbook, only static figures for each time can be displayed. By employing the animation capability of Mathematica, time-dependent behavior will be captured. For simplicity, only one-dimensional wave problem is considered here.

\section{FORMULATION OF ONE-DIMENSIONAL WAVE PROBLEMS}

\section{An Infinite String With a Mass, Spring, and Damper at the Origion}

The governing equation for the one-dimensional wave equation of an infinite string is

$$
c^{2} \frac{\partial^{2} u(x, t)}{\partial x^{2}}=\frac{\partial^{2} u(x, t)}{\partial t^{2}}, \quad-\infty<x<\infty
$$

where $c$ and $u(x, t)$ denote the wave velocity and displacement, respectively. The initial displacement $\phi(x)$ and velocity $\varphi(x)$ conditions are

$$
\begin{gathered}
u(x, 0)=\phi(x) \\
\frac{\partial u(x, 0)}{\partial t}=\varphi(x)
\end{gathered}
$$

By employing the method of characteristic line, we can assume the solution of wave equation as

$$
u(x, t)=P(x+c t)+Q(x-c t)
$$

where $P(x+c t)$ and $Q(x-c t)$ are functions to match initial conditions of Equations (2) and (3). The functions $P(x+c t)$ and $Q(x-c t)$ represent an outgoing-traveling wave and an incoming-traveling wave, respectively. By satisfying Equations (2) and (3) for Equation (4), we obtain the D'Alembert solution

$$
u(x, t)=\frac{1}{2}[\phi(x+c t)+\phi(x-c t)]+\frac{1}{2 c} \int_{x-c t}^{x+c t} \varphi(s) \mathrm{d} s
$$

The solution of the one-dimensional wave equation has two groups of characteristic lines from Equation (5), which can construct a parallelogram in the plane of space and time as shown in Figure 1. By utilizing the D'Alembert's solution, we can obtain the relationship of displacement of four vertexes, which is coined the diamond rule as shown below:

$$
u_{\mathrm{A}}+u_{\mathrm{C}}=u_{\mathrm{B}}+u_{\mathrm{D}}
$$

where $u_{\mathrm{A}}, u_{\mathrm{B}}, u_{\mathrm{C}}$, and $u_{\mathrm{D}}$ denote the displacement of the four points, $\mathrm{A}, \mathrm{B}, \mathrm{C}$, and $\mathrm{D}$, respectively. In order to solve an infinite string with a spring, mass, and damper at the origion, either the Laplace transform or the convolution theorem can be used to derive the exact solution. By using the method of the characteristic line, the domain can be decomposed into four regions. The diagram is shown in Figure 2. By utilizing the diamond rule, the solution of each region can be obtained, which is shown below:

$$
u_{\mathrm{I}}(x, t)=\frac{1}{2}\left[\phi\left(x-c_{1} t\right)+\phi\left(x+c_{1} t\right)\right], \quad(x, t) \in \mathrm{I}
$$

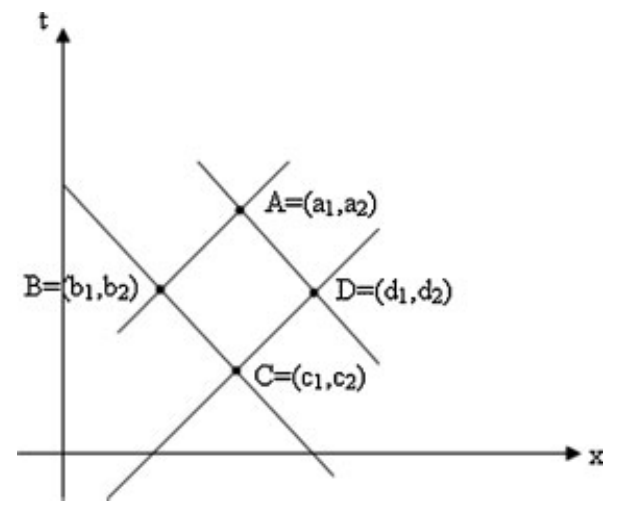

Figure 1 Diamond rule $u(A)+u(C)=u(B)+u(D)$. 


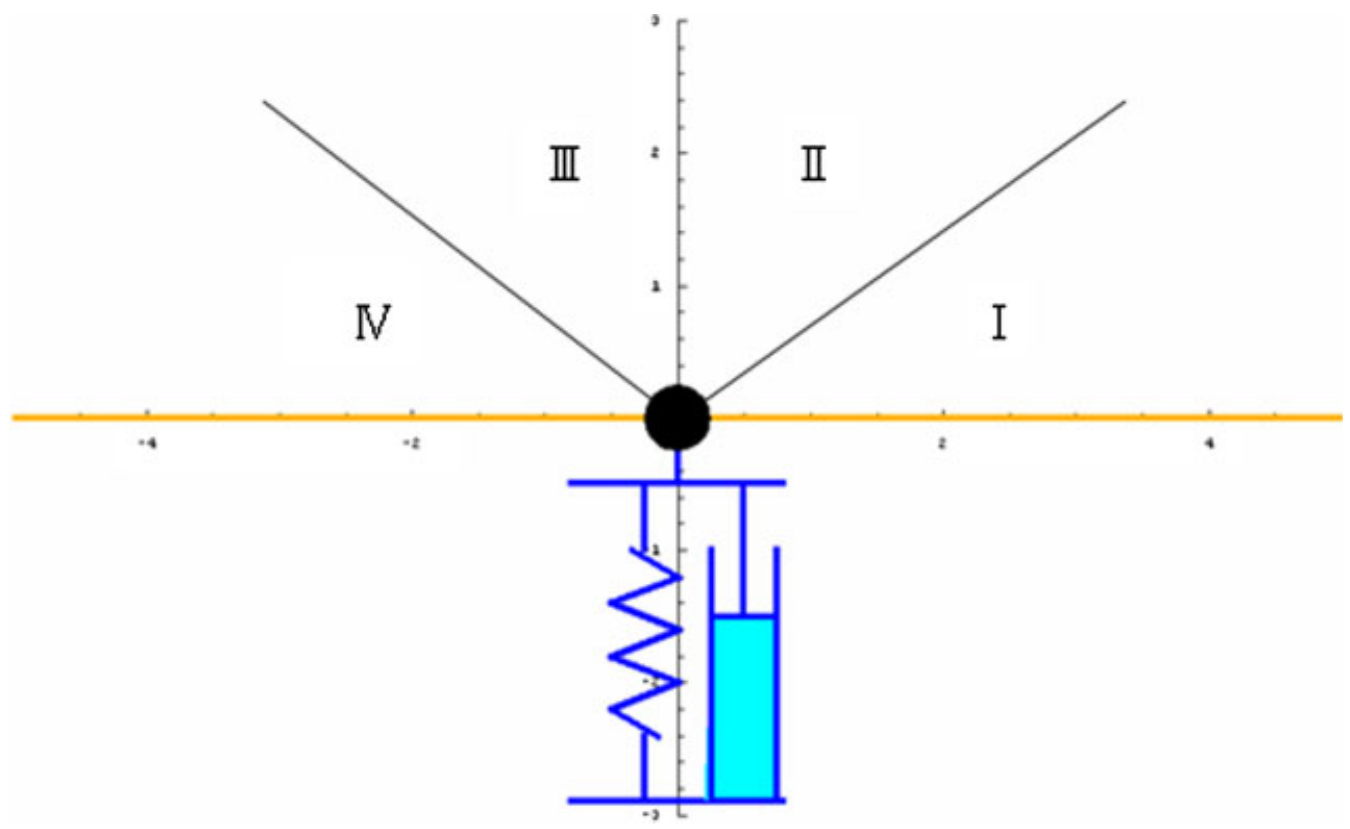

Figure 2 An infinite string with a mass, spring, and damper at the origin.

$$
\begin{aligned}
u_{\mathrm{II}}(x, t)= & \frac{1}{2}\left[\phi\left(x+c_{1} t\right)-\phi\left(-x+c_{1} t\right)\right] \\
& +r\left(t-\frac{x}{c_{1}}\right), \quad(x, t) \in \mathrm{II} \\
u_{\mathrm{III}}= & r\left(t+\frac{x}{c_{2}}\right), \quad(x, t) \in \mathrm{III} \\
& u_{\mathrm{IV}}=0, \quad(x, t) \in \mathrm{IV}
\end{aligned}
$$

where $r(t)$ denotes the displacement of $u(0, t)$. The function $r(t)$ can be obtained by matching the condition of force equilibrium, which is

$\left.T_{\mathrm{II}} \frac{\partial u_{\mathrm{II}}}{\partial x}\right|_{x=0^{+}}-\left.T_{\mathrm{III}} \frac{\partial u_{\mathrm{III}}}{\partial x}\right|_{x=0^{-}}=m \ddot{r}(t)+\xi \dot{r}(t)+k r(t)$

where $m, \xi$, and $k$ denote the constants of mass, damping coefficient and spring constant, respectively, while $T$ denote the string tension $\left(T_{\mathrm{II}}=\rho_{1} c_{1}^{2}, T_{\mathrm{III}}=\rho_{2} c_{2}^{2}\right)$. By using the Laplace transform, Equation (11) can be transformed into

$$
R(s)=\frac{\rho_{1} c_{1}^{2} s}{\left[m s^{2}+\left(\rho_{1} c_{1}+\rho_{2} c_{2}+\xi\right) s+k\right]} \Phi(s)
$$

where $R(s)$ and $\Phi(s)$ are the Laplace transforms of $r(t)$ and $\phi\left(c_{1} t\right)$, respectively. The exact solution $r(t)$ can be obtained by using either the Laplace transform in the $s$ domain or the convolution theorem in the time domain together with Mathematica.

\section{A Semi-Finite String With a Mass, Spring, and Damper at the End}

The governing equation is the same with Equation (1), but the solution domain is $0<x<\infty$. The initial displacement and velocity are the same with Equations (2) and (3), respectively. By using the method of the characteristic line, the domain can be decomposed into two regions as shown in Figure 3, where the solution of each region of the semi-finite string can be obtained by employing the diamond rule, which is shown below:

$$
u_{\mathrm{I}}(x, t)=\frac{1}{2}[\phi(x-c t)+\phi(x+c t)], \quad(x, t) \in \mathrm{I}
$$

$$
\begin{aligned}
& u_{\mathrm{II}}(x, t)=\frac{1}{2}[\phi(x+c t) \\
& \quad-\phi(-x+c t)]+r\left(t-\frac{x}{c}\right), \quad(x, t) \in \mathrm{II}
\end{aligned}
$$

where $r(t)$ denotes the displacement of $u(0, t)$. The function of $r(t)$ can be determined by employing the condition of force equilibrium, which is

$$
\left.T \frac{\partial u}{\partial x}\right|_{x=0^{+}}=m \ddot{r}(t)+\xi \dot{r}(t)+k r(t)
$$




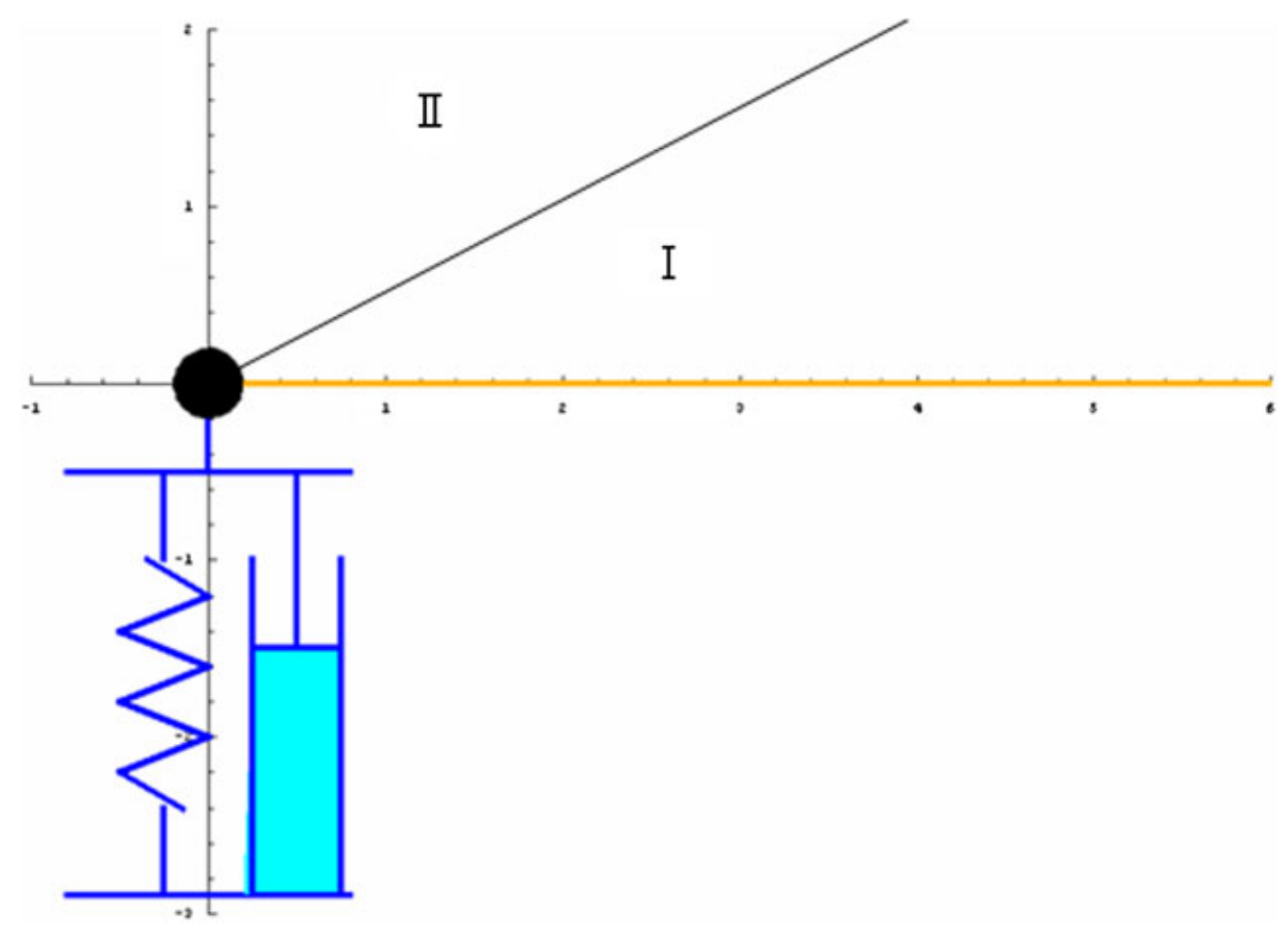

Figure 3 A semi-finite string with a mass, spring, and damper at the end.

By using Laplace transform, Equation (15) can be transformed into

$$
R(s)=\frac{\rho c^{2} s}{\left[m s^{2}+(\rho c+\xi) s+k\right]} \Phi(s)
$$

where $R(s)$ and $\Phi(s)$ are the Laplace transforms of $r(t)$ and $\phi(c t)$. The exact solution of $r(t)$ can be obtained by using either the Laplace transform or the convolution theorem together with Mathematica.

\section{A Finite String}

We employ three methods to solve initial boundary value problems (IBVPs) (i.e., fixed or free), such as the diamond rule, the image method and the series solution. The governing equation is the same with Equation (1), but the domain is $0<x<l$. The initial displacement and velocity are the same with Equations (2) and (3), respectively. The boundary conditions $(\mathrm{BC})$ are shown below:

$$
u(0, t)=u(l, t)=0
$$

The derivations for analytical solutions using the three different methods are shown as follows.

Diamond Rule. By using the method of characteristic line, we can decompose the space- time $(x, t)$ plane into several regions as shown in Figure 4. The solution of each region can be obtained by using the diamond rule, which is shown below:

$$
\begin{gathered}
u_{\mathrm{I}}(x, t)=\frac{1}{2}[\phi(x+c t)+\phi(x-c t)], \\
u_{\mathrm{II}}(x, t)=\frac{1}{2}[\phi(x+c t)-\phi(-x+c t)], \\
u_{\mathrm{III}}(x, t)=\frac{1}{2}[\phi(x-c t)-\phi(2 l-(x+c t))],
\end{gathered}
$$

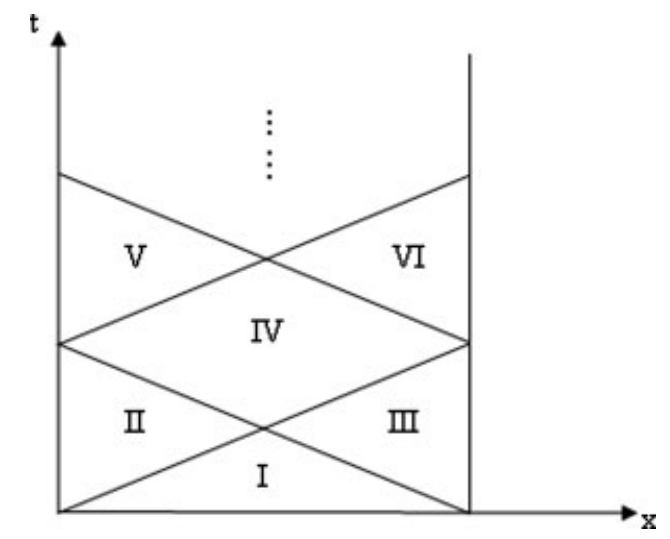

Figure 4 Solution for each region using the diamond rule. 


$$
\begin{aligned}
u_{\mathrm{IV}}(x, t)= & -\frac{1}{2}[\phi(-x+c t) \\
& +\phi(2 l-(-x+c t))] \ldots
\end{aligned}
$$

The complete solution can be obtained by successively employing the diamond rule.

Image Method. For a finite string, we employ the image method to handle the wave problem with fixed or free boundary. In order to satisfy the fixed or free $\mathrm{BC}$, we introduce an anti-symmetrical and a symmetrical source, respectively. According to Huygen's principle, we use the image wave to match the $\mathrm{BC}$. Therefore, the finite string is imbedded into an infinite string by using the image method as shown in Figure 5. The solution can be obtained by utilizing D'Alembert solution for the infinite string. We obtain the solution by selecting the finite domain $(0<x<l)$ from the infinite string.

Series Solution. The series representation for the displacement can be written as

$$
u(x, t)=\sum_{n=1}^{\infty} T_{n}(t) X_{n}(x), \quad 0<x<l
$$

where $l$ is the length of the string, $T_{n}(t)$ is the generalized coordinate, and $X_{n}(x)$ is the nth modal shape satisfying the orthogonal property:

$$
\int_{0}^{l} X_{m}(x) X_{n}(x) \mathrm{d} x=\left\{\begin{array}{l}
0, \text { if } n \neq m \\
N_{n}, \text { if } n=m
\end{array}\right.
$$

The generalized coordinate can be written as

$$
T_{n}(t)=\frac{1}{N_{n}} \int_{0}^{l} u(x, t) X_{n}(x) \mathrm{d} x
$$

By assuming the solution to be in a series form, we have

$$
u(x, t)=\sum_{n=1}^{\infty}\left(d_{n} \cos \left[\frac{c n \pi}{l} t\right]+e_{n} \sin \left[\frac{c n \pi}{l} t\right]\right) \sin \left[\frac{n \pi x}{l}\right]
$$

where the unknown coefficients can be determined by

$$
d_{n}=\frac{2}{l} \int_{0}^{l} \phi(x) \sin \left[\frac{n \pi x}{l}\right] \mathrm{d} x
$$

$$
e_{n}=\frac{2}{c n \pi} \int_{0}^{l} \varphi(x) \sin \left[\frac{n \pi x}{l}\right] \mathrm{d} x
$$

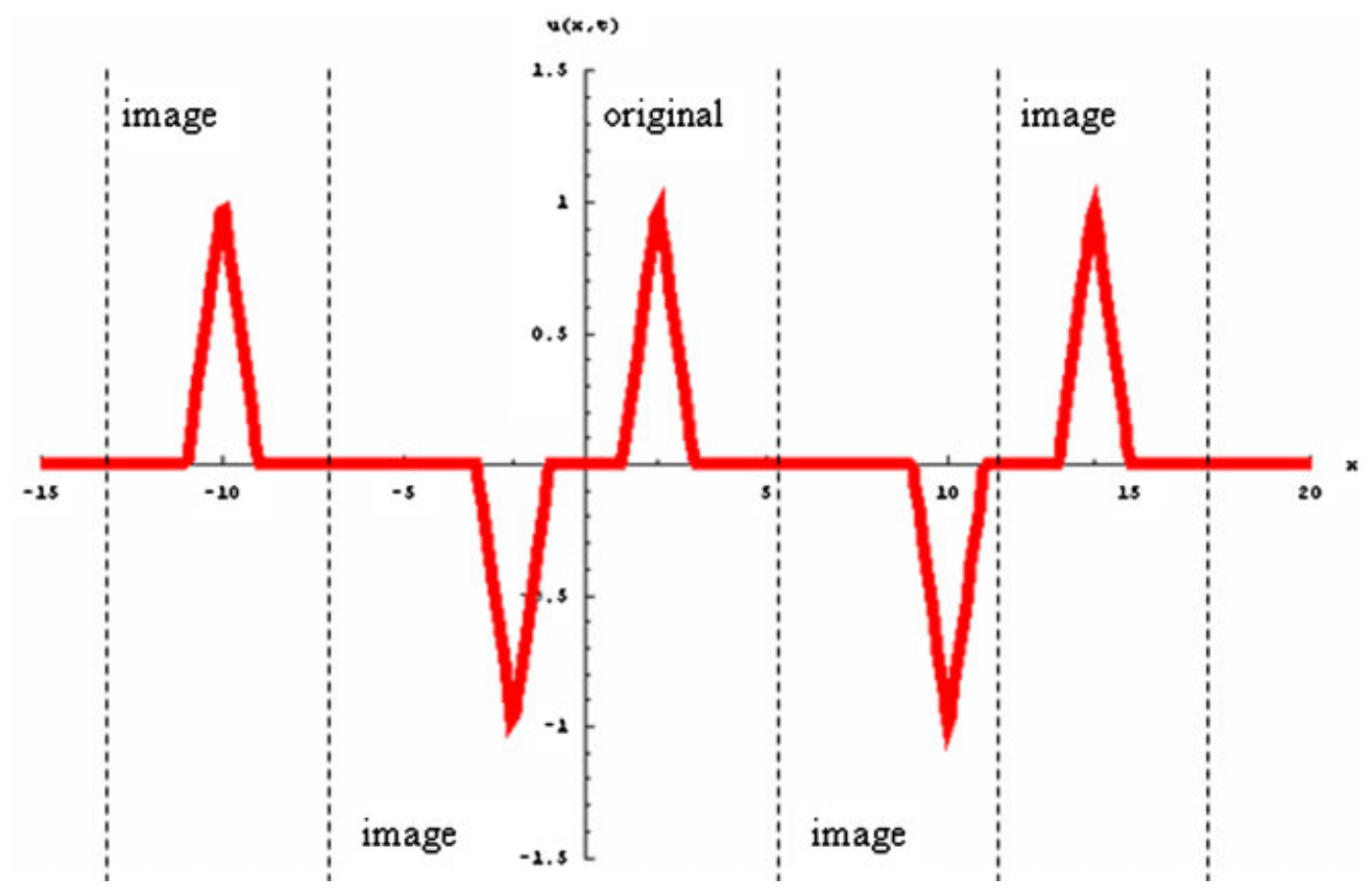

Figure 5 Diagram using the image method. 


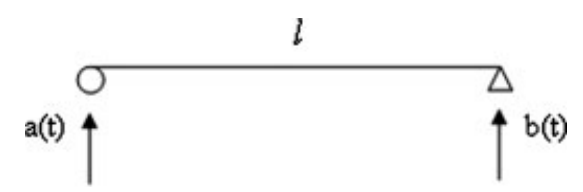

Figure 6 A fixed string subjected to support motions.

\section{A String Subjected to Support Motions}

A string subjected to support motions is considered in this subsection as shown in Figure 6. The governing equation is the same with Equation (1), but the solution domain is $0<x<l$. The initial displacement and velocity are the same with Equations (2) and (3), respectively, but the initial displacement is null $(\phi(x)=0)$ and the initial velocity is at rest $(\varphi(x)=0)$. The BCs of support motions are

$$
\begin{aligned}
& u(0, t)=a(t) \\
& u(l, t)=b(t)
\end{aligned}
$$

where $a(t)$ and $b(t)$ are the support motions. In order to transform the non-homogenous $\mathrm{BC}$ of the support to be homogenous, the solution can be decomposed into two parts by using the quasi-static decomposition technique:

$$
u(x, t)=U(x, t)+\sum_{n=1}^{\infty} T_{n}(t) X_{n}(x), \quad 0<x<l
$$

where $l$ is the length of the string or beam, $U(x, t)$ is the quasi-static solution, $T_{n}(t)$ is the generalized coordinate, and $X_{n}(x)$ is the modal shape satisfying the orthogonal property,

$$
\int_{0}^{l} X_{\mathrm{m}}(x) X_{n}(x) \mathrm{d} x=\left\{\begin{array}{l}
0, \text { if } n \neq m \\
N_{n}, \text { if } n=m
\end{array}\right.
$$

The generalized coordinate can be determined by

$$
T_{n}(t)=\frac{1}{N_{n}} \int_{0}^{l} u(x, t) X_{n}(x) \mathrm{d} x
$$

The quasi-static part $U(x, t)$ in Equation (30) must satisfy

$$
\frac{\partial^{2} U(x, t)}{\partial x^{2}}=0, \quad 0<x<l
$$

and is subject to the following BCs:

$$
U(0, t)=a(t), \quad U(l, t)=b(t)
$$

By solving Equation (33) subject to BCs of Equation
(34), we have

$$
U(x, t)=a(t)\left(1-\frac{x}{l}\right)+b(t) \frac{x}{l}
$$

By using the approach of quasi-static decomposition technique [3], we have

$$
\begin{aligned}
u(x, t)= & \sum_{n=1}^{\infty}\left\{\frac{2 l}{c n^{2} \pi^{2}} \sin \left[\frac{c n \pi}{l} t\right]\left[b^{\prime}(0)(-1)^{n}-a^{\prime}(0)\right]\right. \\
& +\frac{2}{n \pi}\left[b(0)(-1)^{n}-a(0)\right] \cos \left[\frac{c n \pi}{l} t\right] \\
+ & \frac{2 l}{c n^{2} \pi^{2}} \int_{0}^{t} \sin \left[\frac{c n \pi}{l}(t-\tau)\right]\left[b^{\prime \prime}(\tau)(-1)^{n}\right. \\
& \left.\left.-a^{\prime \prime}(\tau)\right] \mathrm{d} \tau\right\} \sin \left(\frac{n \pi x}{l}\right)+U(x, t)
\end{aligned}
$$

\section{A Beam Subjected to Support Motions}

The seismic response of a Rayleigh-damped Bernoulli-Euler beam subjected to multi-support motions as shown in Figure 7 can be described by the following governing equation:

$$
\begin{gathered}
\rho A \ddot{u}(x, t)+\left(2 \alpha \rho A+\beta E I \frac{\partial^{4}}{\partial x^{4}}\right) \dot{u}(x, t) \\
+E I \frac{\partial^{4} u(x, t)}{\partial x^{4}}=f(x, t), \quad 0<x<l
\end{gathered}
$$

where the superimposed dot denotes a time derivative, $E I, \rho \mathrm{A}$, and $l$ denote the flexural rigidity, mass per unit length and length of the single-span beam of the bridge, $\alpha$ and $\beta$ are the coefficients of Rayleigh damping. The initial displacement is null $(\phi(x)=0)$ and the initial velocity is at rest $(\varphi(x)=0)$. The BCs of support motions are the same with Equations (28) and (29). Other BCs are shown below:

$$
\frac{\partial^{2} u(0, t)}{\partial x^{2}}=\frac{\partial^{2} u(l, t)}{\partial x^{2}}=0
$$

The displacement of $u(x, t)$ is a time-space function. The solution can be decomposed into two parts by using the concept of quasi-static and dynamic solution

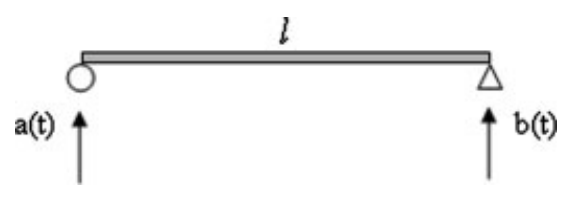

Figure 7 A simply supported beam subjected to support motions. 
as shown below:

$$
u(x, t)=U(x, t)+\sum_{n=1}^{\infty} T_{n}(t) X_{n}(x), \quad 0<x<l
$$

The quasi-static part $u(x, t)$ must satisfy

$$
\frac{\partial^{4} U(x, t)}{\partial x^{4}}=0
$$

and the support motions are the same with Equation (34). The BCs are shown below:

$$
\frac{\partial^{2} U(0, t)}{\partial x^{2}}=\frac{\partial^{2} U(l, t)}{\partial x^{2}}=0
$$

By solving Equation (40) subject to BCs of Equations (34) and (41), we have

$$
U(x, t)=a(t)\left(1-\frac{x}{l}\right)+b(t) \frac{x}{l}
$$

The $n$th natural mode $X_{n}(\mathrm{x})$ of the eigen-system is

$$
X_{n}(x)=\sin \left(\frac{n \pi x}{l}\right), \quad n=1,2, \ldots
$$

and the corresponding natural frequencies $\omega_{n}$ are

$$
\omega_{n}=\left(\frac{n \pi}{l}\right)^{2} \sqrt{\frac{E I}{\rho A}}, \quad n=1,2, \ldots
$$

Substituting Equation (39) into Equation (37), we obtain

$$
\begin{aligned}
\sum_{n=1}^{\infty} \rho\left[\ddot{T}_{n}(t)\right. & \left.+2 \xi_{n} \omega_{n} \dot{T}_{n}(t)+\omega_{n}^{2} q_{n}(t)\right] X_{n}(x) \\
= & -\rho \ddot{U}(x, t)-2 \alpha \rho \dot{U}(x, t)
\end{aligned}
$$

where the $n$th damping ratio $\xi_{n}$ is defined by

$$
2 \xi_{n} \omega_{n}=2 \alpha+\beta \omega_{n}^{2}
$$

By employing the orthogonal condition of Equation (23), $T_{n}(t)$ must satisfy the following second order ordinary differential equation (ODE):

$$
\ddot{T}_{n}(t)+2 \xi_{n} \omega_{n} \dot{T}_{n}(t)+\omega_{n}^{2} q_{n}(t)=\frac{\ddot{F}_{n}(t)}{N}+\frac{2 \alpha \dot{F}_{n}(t)}{N}
$$

where $N=\rho \mathrm{l} / 2$ and

$$
F_{n}(t)=-\int_{0}^{l} \rho U(x, t) X_{n}(x) \mathrm{d} x
$$

After considering the initial conditions $(\phi(x)=0$ and $\varphi(x)=0)$, we have

$$
N T_{n}(0)=-\int_{0}^{l} \rho U(x, t) X_{n}(x) \mathrm{d} x=F_{n}(0)
$$

$$
N \dot{T}_{n}(0)=-\int_{0}^{l} \rho \dot{U}(x, t) X_{n}(x) \mathrm{d} x=\dot{F}_{n}(0)
$$

Thus, we can solve for $T_{n}(t)$ according to Equations (47), (49) and (50), that is

$$
\begin{aligned}
& T_{n}(t)=\frac{F_{n}(t)}{N}-\frac{1}{N \omega_{n} \sqrt{1-\xi_{n}^{2}}} \int_{0}^{t} \mathrm{e}^{-\xi_{n} \omega_{n}(t-\tau)} \\
& \sin \left(\omega_{n}^{d}(t-\tau)\right)\left[\omega_{n}^{2} F_{n}(\tau)+\beta \dot{F}_{n}(\tau)\right] \mathrm{d} \tau
\end{aligned}
$$

where $\omega_{n}^{d}=\omega_{n} \sqrt{1-\xi_{n}^{2}}$ is the $n$th damping frequency. Then, the series solutions for the displacement is

$$
u(x, t)=U(x, t)+\sum_{n=1}^{\infty} T_{n}(t) \sin \left(\frac{n \pi x}{l}\right)
$$

where $T_{n}(t)$ is determined by Equation (51).

\section{ILLUSTRATIVE EXAMPLES WITH ANIMATION USING THE MATHEMATICA}

Six examples are utilized to demonstrate the validity of present approach.

\section{Example 1: An Infinite String With a Mass, Spring, and Damper at the Origin}

The first example is an infinite string with a mass, spring, and damper at the origin as shown in Figure 2. The initial conditions are

$$
\begin{aligned}
& u(x, 0)=\phi(x)= \begin{cases}\sin (x), 2 \pi \leq x \leq 3 \pi \\
0, & \text { otherwise }\end{cases} \\
& \dot{u}(x, 0)=\varphi(x)=0
\end{aligned}
$$

The input data are as follows:

$c=1 \mathrm{~m} / \mathrm{s}, \rho=1 \mathrm{~kg} / \mathrm{m}, m=1 \mathrm{~kg}, k=1 \mathrm{~N} / \mathrm{m}, \xi=0.1$

where $c$ and $\rho$ are the wave velocity and the density of a unit length, respectively. By utilizing the diamond rule, the solution in each region can be expressed below: 


$$
\begin{gathered}
u_{\mathrm{I}}(x, t)=\frac{1}{2}[\sin (x-t)+\sin (x+t)], \quad(x, t) \in \mathrm{I} \\
\begin{aligned}
& u_{\mathrm{II}}(x, t)=\frac{1}{2}[\sin (x+t)-\sin (-x+t)] \\
&+r(t-x), \quad(x, t) \in \mathrm{II} \\
& u_{\mathrm{III}}(x, t)=r(t+x), \quad(x, t) \in \mathrm{III} \\
& u_{\mathrm{IV}}(x, t)=0, \quad(x, t) \in \mathrm{IV}
\end{aligned}
\end{gathered}
$$

where $r(t)=u(0, t)$ is the time history of the unknown displacement at the origin. To match the condition of force equilibrium, we have

$$
\phi(t)-2 r^{\prime}(t)=m \ddot{r}(t)+\xi \dot{r}(t)+k r(t)
$$

after substituting $\rho=1(\mathrm{~kg} / \mathrm{m})$ and $c=1(\mathrm{~m} / \mathrm{s})$. By employing either the Laplace transform or the convolution theorem using Mathematica, we can solve $r(t)$. The diagram of the solution is shown in Figure $8 \mathrm{a}$ and $\mathrm{b}$.

\section{Example 2: Two Semi-Infinite Strings Joined at Origin}

The second example is an infinite string with two media separated at the origin as shown in Figure 9. The initial conditions are the same with Equation (53). The input data are as follows: $c_{1}=1 \mathrm{~m} / \mathrm{s}, c_{2}=2 \mathrm{~m} / \mathrm{s}, \rho_{1}=1 \mathrm{~kg} / \mathrm{m}, \rho_{2}=2 \mathrm{~kg} / \mathrm{m}$, where $c$ and $\rho$ are the wave velocity and the density of an unit length, respectively. By utilizing the diamond rule, the solution in each region can be expressed below: a
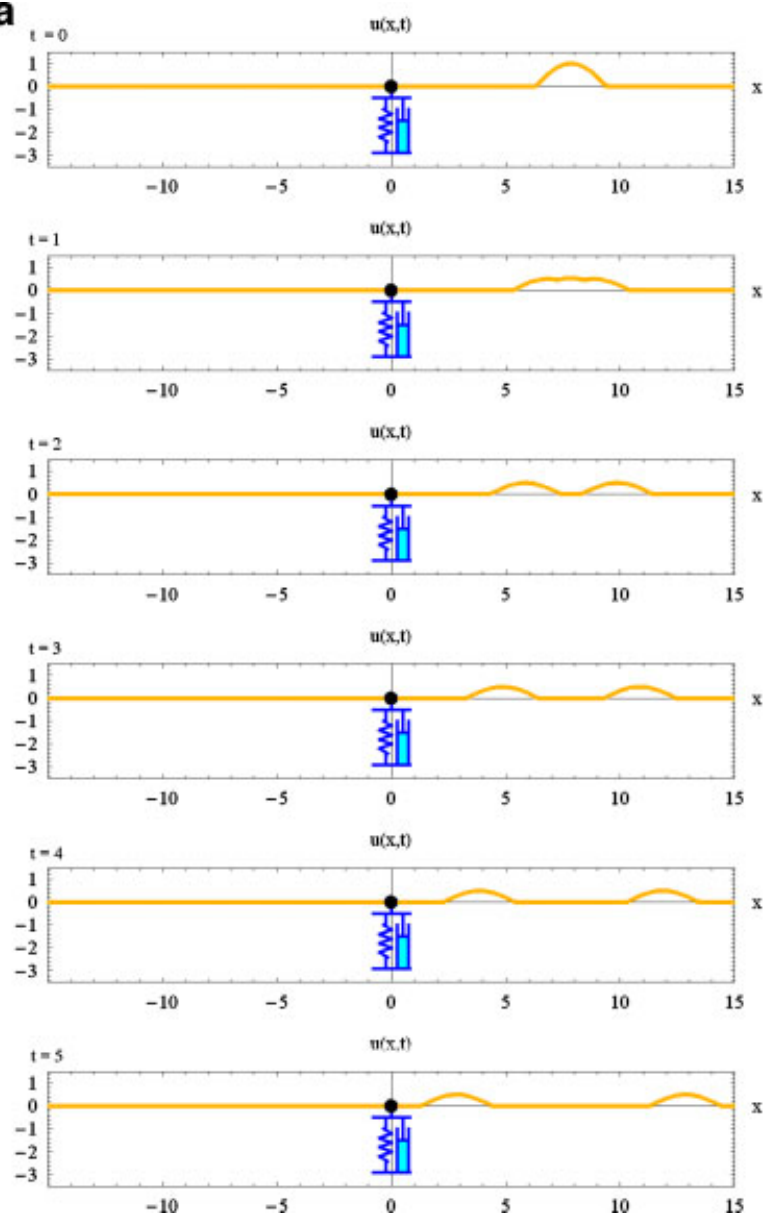

b
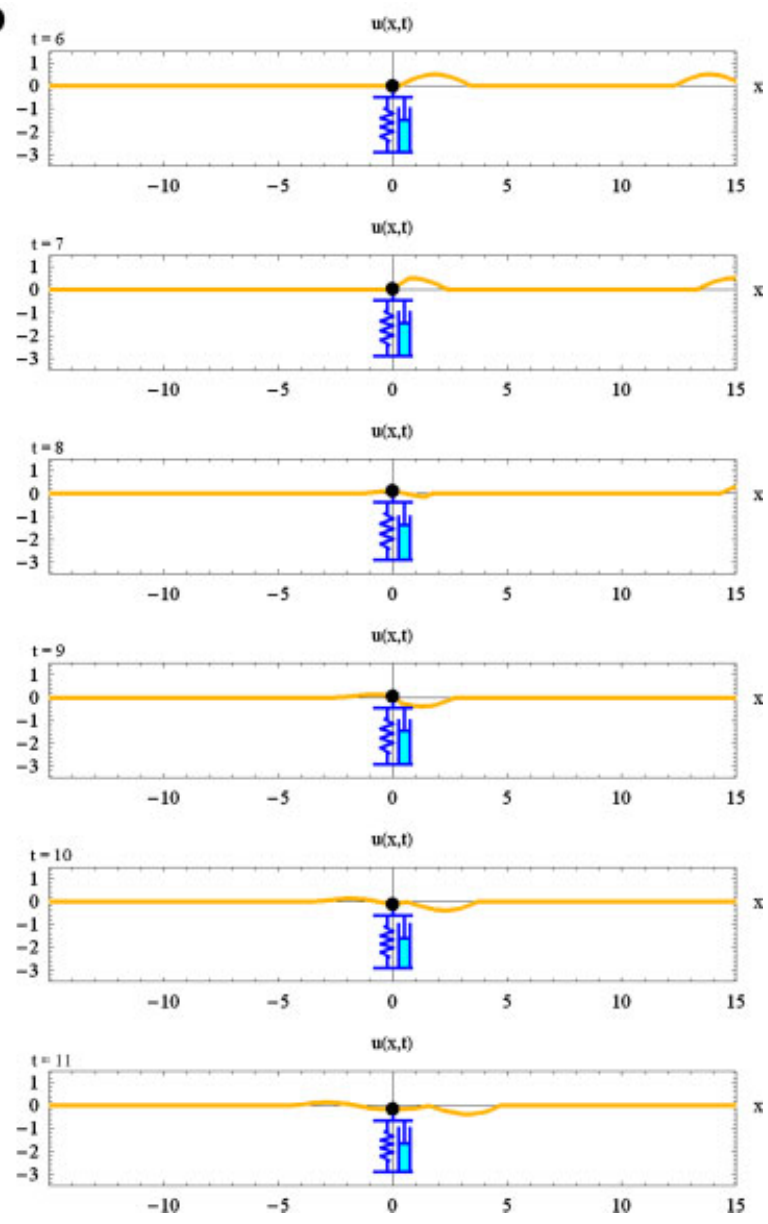

Figure 8 (a) Diagram of the solution of an infinite string $(t=0-5)$. (b) Diagram of the solution of an infinite string $(\mathrm{t}=6-11)$. 


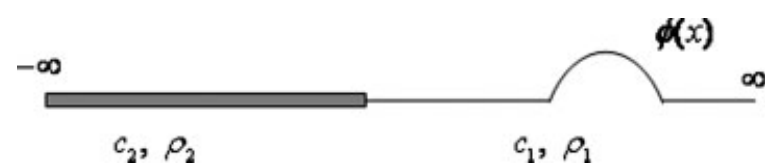

Figure 9 An infinite string with two-media.

$$
\begin{gathered}
u_{\mathrm{I}}(x, t)=\frac{1}{2}[\sin (x-t)+\sin (x+t)], \quad(x, t) \in \mathrm{I} \\
u_{\mathrm{II}}(x, t)=\frac{1}{2}[\sin (x+t)-\sin (-x+t)]+r(t-x), \\
(x, t) \in \mathrm{II} \\
u_{\mathrm{III}}(x, t)=r\left(t+\frac{x}{2}\right), \quad(x, t) \in \mathrm{III} \\
u_{\mathrm{IV}}(x, t)=0, \quad(x, t) \in \mathrm{IV}
\end{gathered}
$$

where $r(t)=u(0, t)$ is the time history of the unknown displacement at the origin. To match the condition of force equilibrium, we have

$$
r(t)=\frac{1}{5} \phi(t)
$$

after substituting $c_{1}=1 \mathrm{~m} / \mathrm{s}, c_{2}=2 \mathrm{~m} / \mathrm{s}, \rho_{1}=1 \mathrm{~kg} /$ $\mathrm{m}, \rho_{2}=2 \mathrm{~kg} / \mathrm{m}$. Then we can obtain the function of $r(t)$. The diagram of the solution is shown in Figure 10a and $\mathrm{b}$. For the parameter study of impendence ratio, another case of ratio $\rho_{1} c_{1} / \rho_{2} c_{2}=$ 10 is also plotted in Figure 11a and b.

\section{Example 3: A Semi-Infinite String With a Mass, Spring, and Damper at the End}

The third example is a semi-infinite string with a mass, spring, and damper at the end. The initial conditions are the same with Equation (53).

The input data are as follows:

$$
c=1 \mathrm{~m} / \mathrm{s}, \rho=1 \mathrm{~kg} / \mathrm{m}, m=1 \mathrm{~kg}, k=1 \mathrm{~N} / \mathrm{m}, \xi=0.2 \text {. }
$$

By utilizing the diamond rule, the solution of each region can be similarly expressed

$$
\begin{gathered}
u_{\mathrm{I}}(x, t)=\frac{1}{2}[\sin (x-t)+\sin (x+t)], \quad(x, t) \in \mathrm{I} \\
u_{\mathrm{II}}(x, t)=\frac{1}{2}[\sin (x-t)-\sin (-x+t)]+r(t-x),
\end{gathered}
$$

$$
(x, t) \in \mathrm{II}
$$

To match the condition of force equilibrium, we have

$$
\phi(t)-r^{\prime}(t)=m \ddot{r}(t)+\xi \dot{r}(t)+k r(t)
$$

By employing either the Laplace transform or the convolution theorem using the Mathematica symbolic software, we can solve the function of $r(t)$, which satisfies the second order ODE. Therefore the diagram of the solution can be shown in Figure 12a and b by employing Mathematica software.

\section{Example 4: A Finite String With Fixed-End Boundary Conditions}

The fourth example is a finite string subject to the fixed-end $\mathrm{BCs}$

$$
\begin{aligned}
& u(0, t)=0 \\
& u(l, t)=0
\end{aligned}
$$

and initial conditions

$$
\begin{aligned}
& u(x, 0)=\phi(x)=\left\{\begin{array}{l}
x-1,1 \leq x<2 \\
3-x, 2 \leq x<3
\end{array}\right. \\
& \dot{u}(x, 0)=\varphi(x)=0
\end{aligned}
$$

The problem can be solved by utilizing the image method. In order to satisfy the BCs, we can obtain the image part, as shown below:

$$
\begin{gathered}
\phi_{1}^{i}(x)=\left\{\begin{array}{l}
-3-x,-3 \leq x<-2 \\
x+1,-2 \leq x<-1,
\end{array}\right. \\
\phi_{2}^{i}(x)=\left\{\begin{array}{l}
9-x, 9 \leq x<10 \\
x-11,10 \leq x<11,
\end{array}\right. \\
\phi_{3}^{i}(x)=\left\{\begin{array}{l}
x+11,-11 \leq x<-10 \\
-9-x,-10 \leq x<-9,
\end{array}\right. \\
\phi_{4}^{i}(x)=\left\{\begin{array}{l}
x-13,13 \leq x<14 \\
15-x, 14 \leq x<15
\end{array}\right.
\end{gathered}
$$

where the superscript $i$ denote the image part and the subscript denotes the number of times for images. Then, we employ the D'Alembert solution for an infinite string to obtain the solution, which is shown in Figure 13a and $b$.

\section{Example 5: A String Subjected to Support Motions}

The fifth example is a string subjected to support motions. The initial conditions are 
a

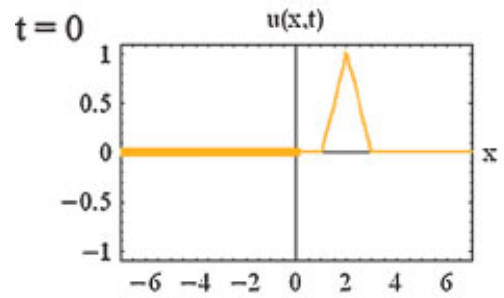

$\mathrm{t}=2$

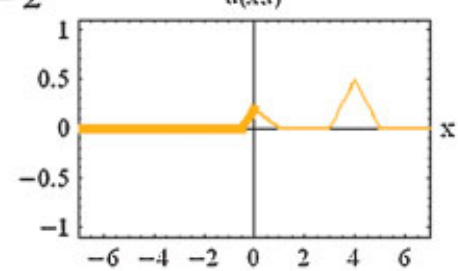

$\mathrm{t}=4$

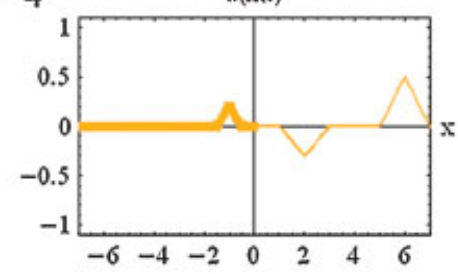

b

$t=6$

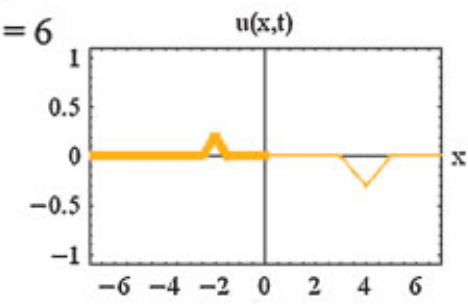

$t=8$

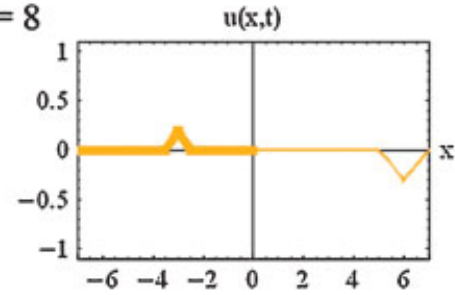

$t=10$

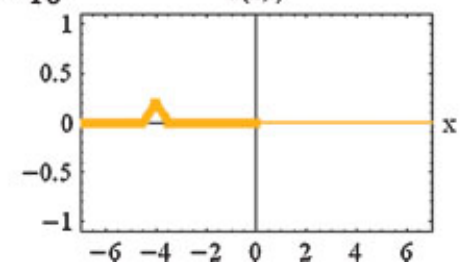

$t=1$

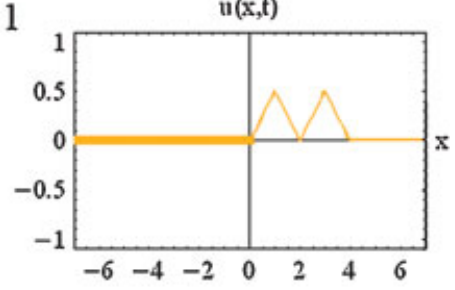

$\mathrm{t}=3$

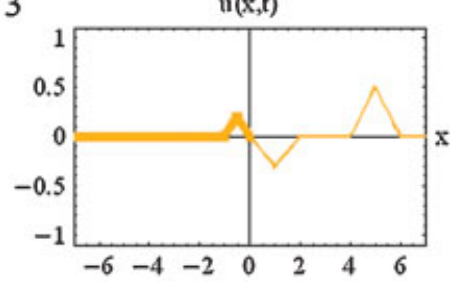

$\mathrm{t}=5$

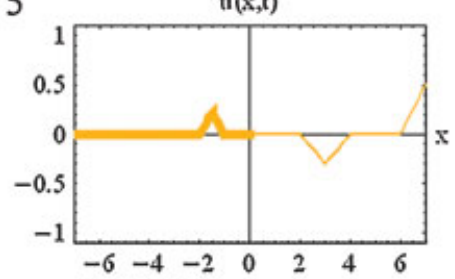

$t=7$

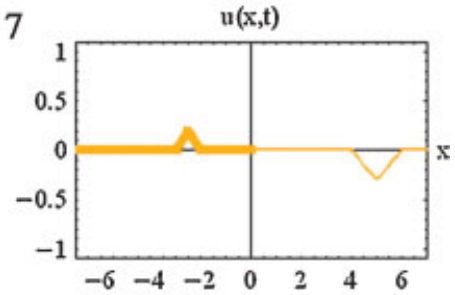

$t=9$

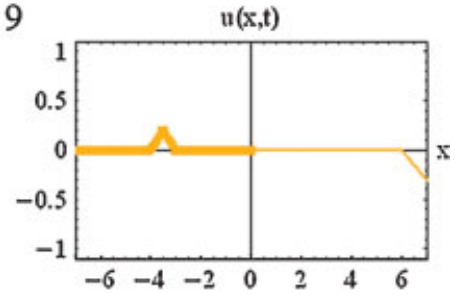

$t=11$

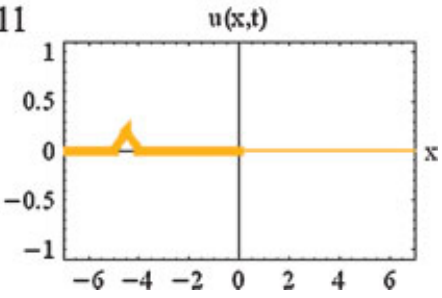

Figure 10 (a) Diagram of solution of an infinite string with two-media $\left(\rho_{2} \mathrm{c}_{2} / \rho_{1} \mathrm{c}_{1}=4\right)$ $(t=0-5)$. (b) Diagram of solution of an infinite string with two-media $\left(\rho_{2} c_{2} / \rho_{1} c_{1}=4\right)$ $(\mathrm{t}=6-11)$. 
a

$\mathrm{t}=0$

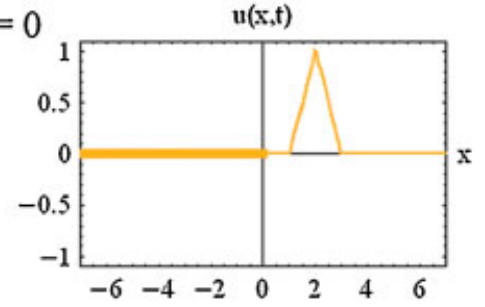

$\mathrm{t}=2$

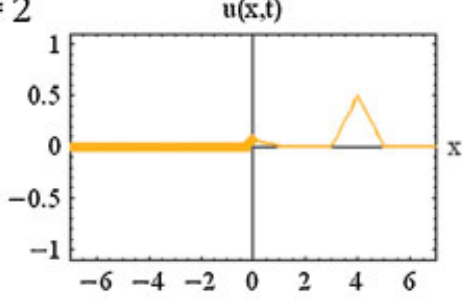

$\mathrm{t}=4$

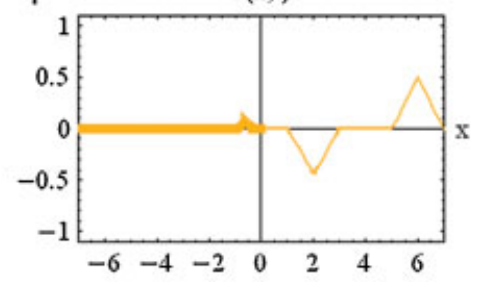

b

$t=6$

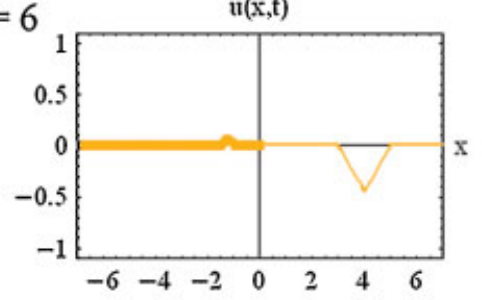

$\mathrm{t}=8$

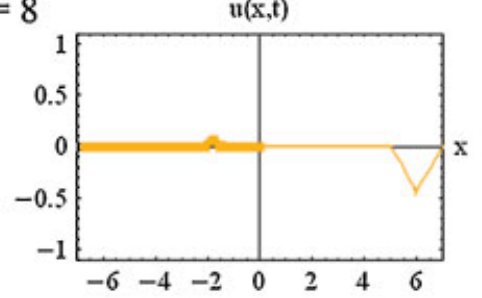

$\mathrm{t}=10$

$\mathrm{u}(\mathrm{x}, \mathrm{t})$

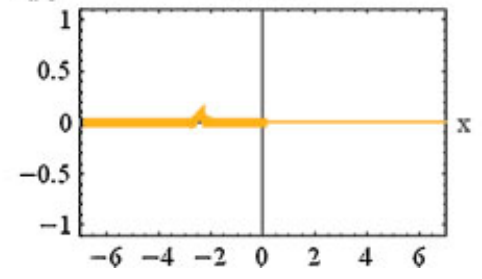

$t=1$

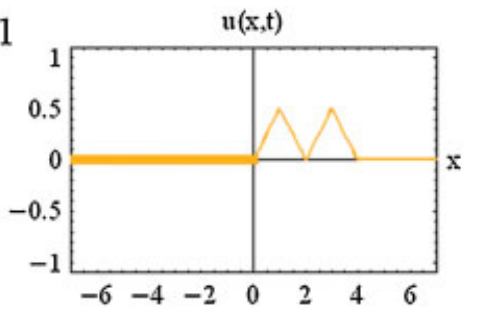

$t=3$

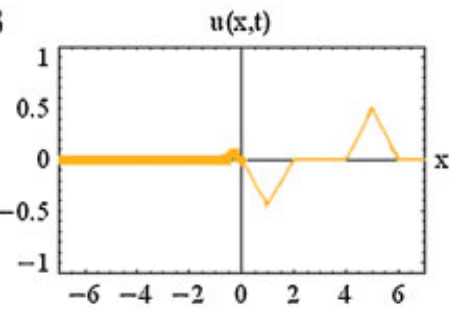

$t=5$

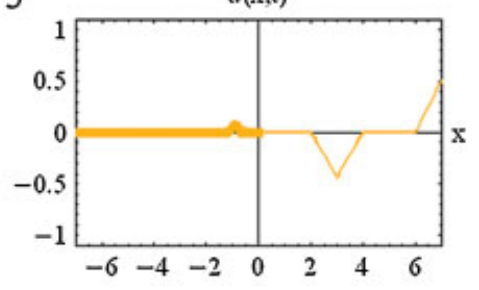

$\mathrm{t}=7$

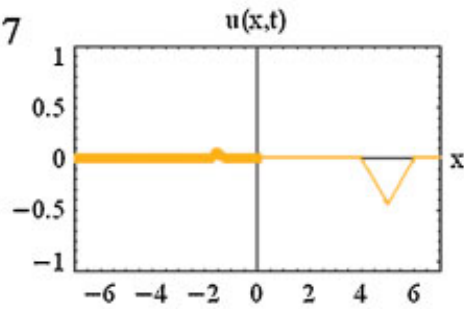

$t=9$

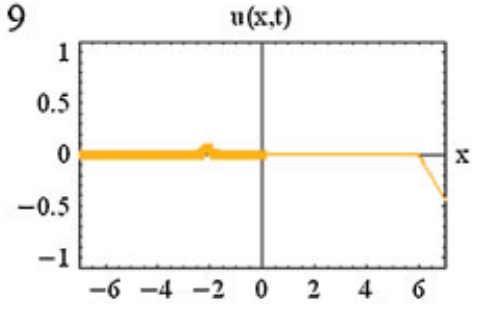

$\mathrm{t}=11$

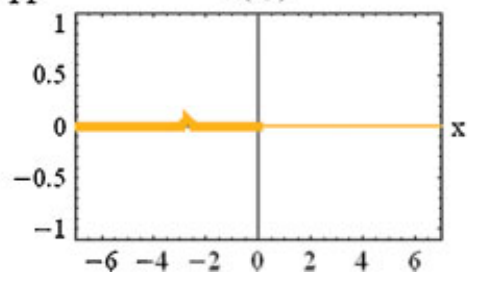

Figure 11 (a) Diagram of solution of an infinite string with two-media $\left(\rho_{2} \mathrm{c}_{2} / \rho_{1} \mathrm{c}_{1}=10\right)$ $(\mathrm{t}=0-5)$. (b) Diagram of solution of an infinite string with two-media $\left(\rho_{2} \mathrm{c}_{2} / \rho_{1} \mathrm{c}_{1}=10\right)$ $(\mathrm{t}=6-11)$. 
a

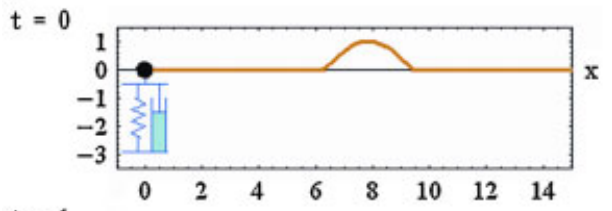

$t=1$

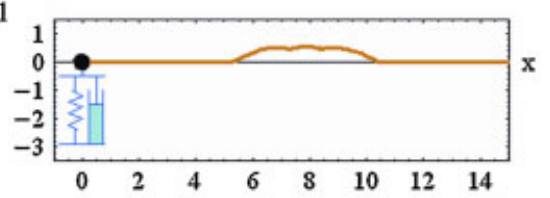

$\mathrm{t}=2$

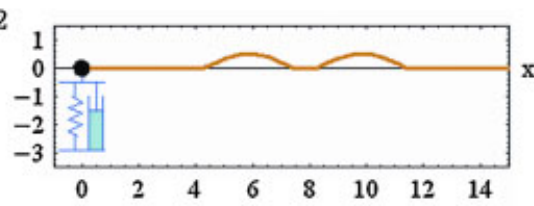

$t=3$

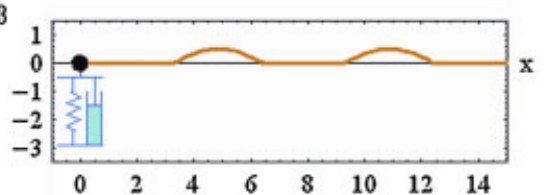

$\mathrm{t}=4$

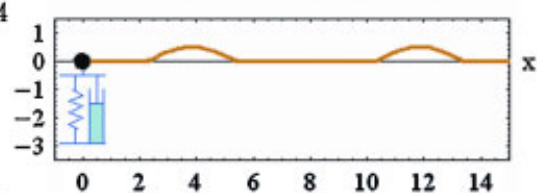

$t=5$

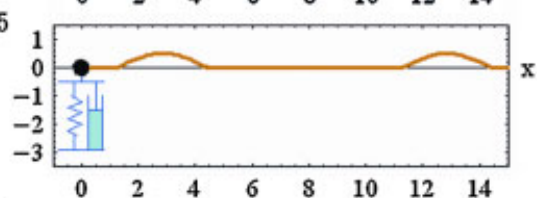

$t=6$

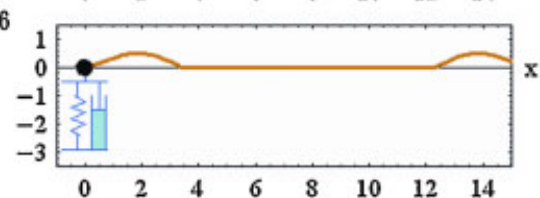

$\mathrm{t}=7$
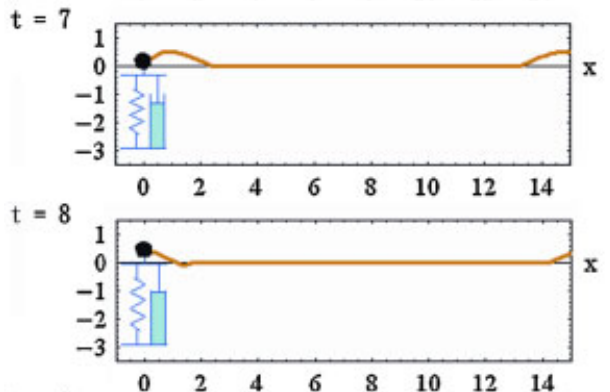

$t=9$

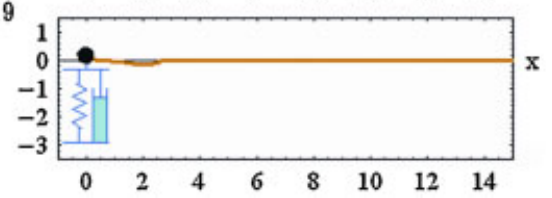

b

$\mathrm{t}=10$

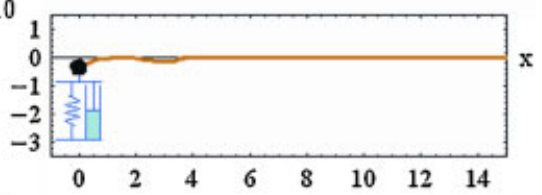

$\mathrm{t}=11$

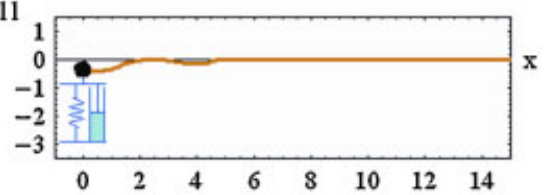

$\mathrm{t}=12$

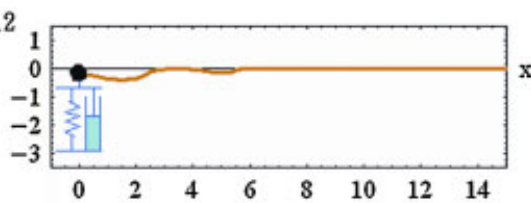

$t=13$

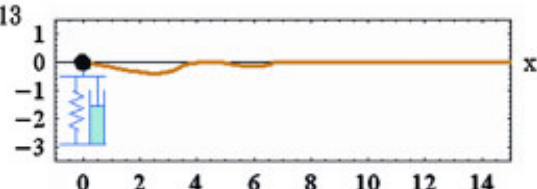

$\mathrm{t}=14$
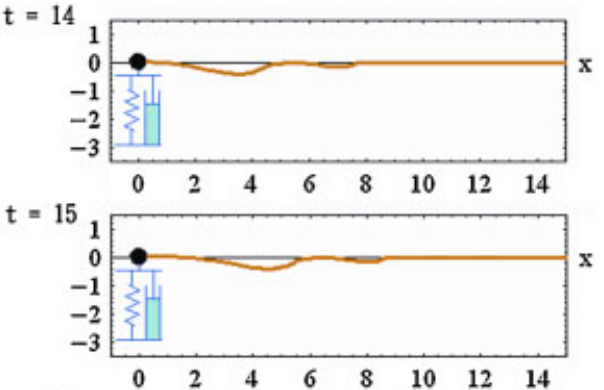

$t=16$

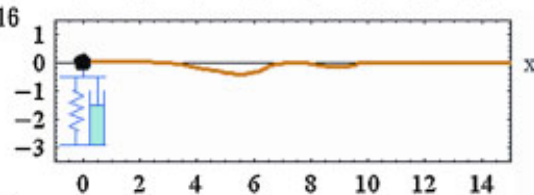

$\mathrm{t}=17$

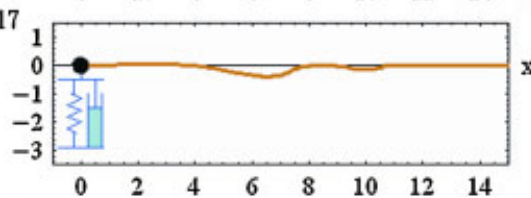

$t=18$
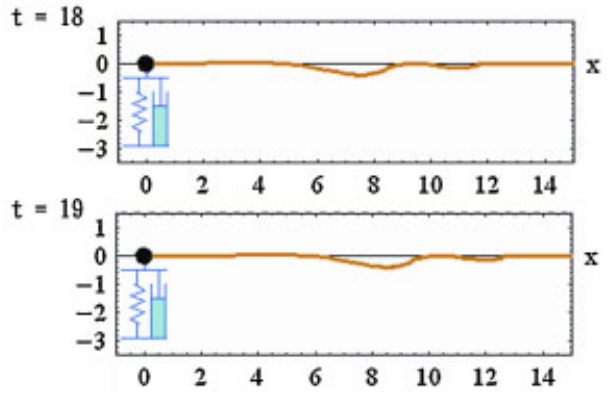

Figure 12 (a) Diagram of the solution of the semi-infinite string $(t=0-9)$. (b) Diagram of the solution of the semi-infinite string $(t=10-19)$. 
a
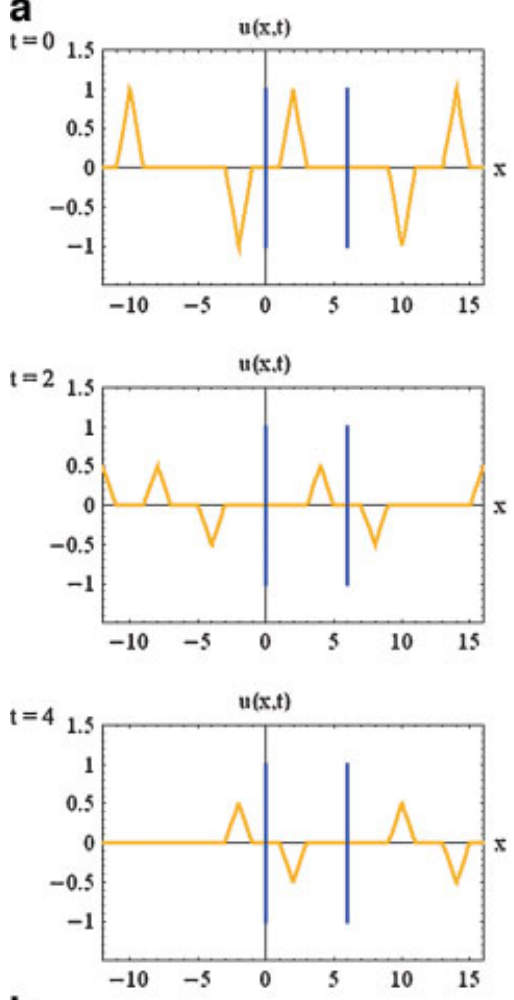

b
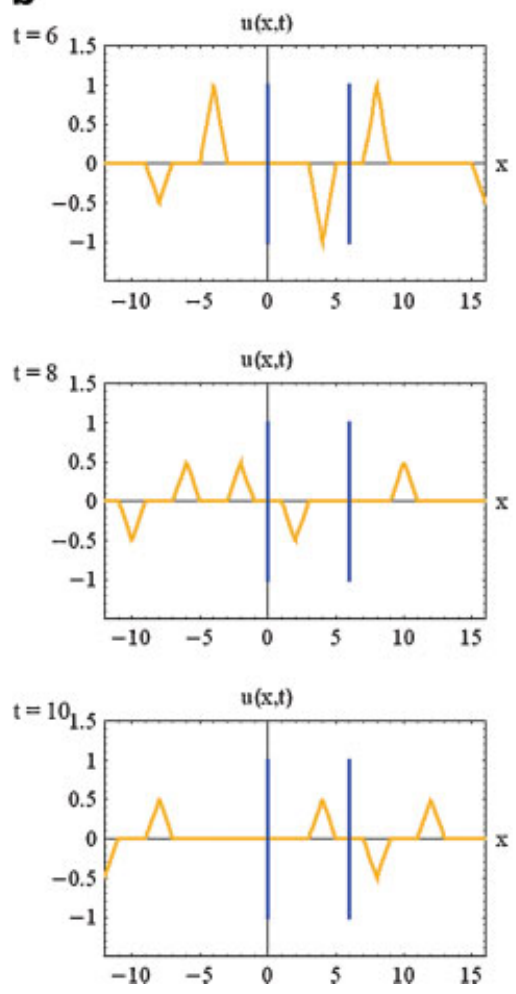
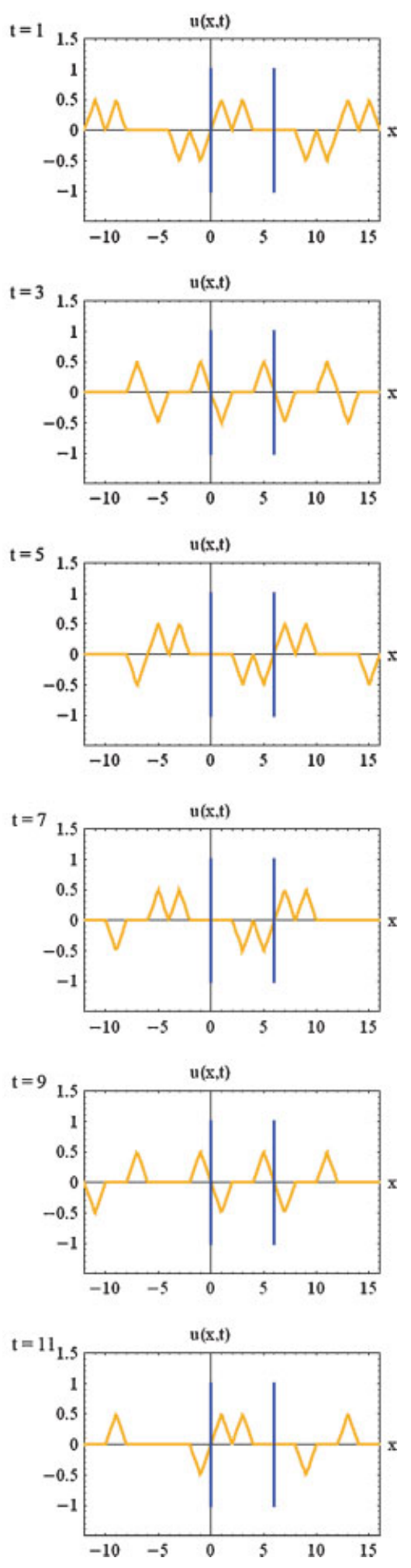

Figure 13 (a) Diagram of the solution of a finite string by using the image method $(t=0-5)$. (b) Diagram of the solution of a finite string by using the image method $(t=6-11)$. 


$$
\begin{aligned}
& u(x, 0)=0,0<x<l \\
& \dot{u}(x, 0)=0,0<x<l
\end{aligned}
$$

and the $\mathrm{BCs}$ of the in-phase support motions are
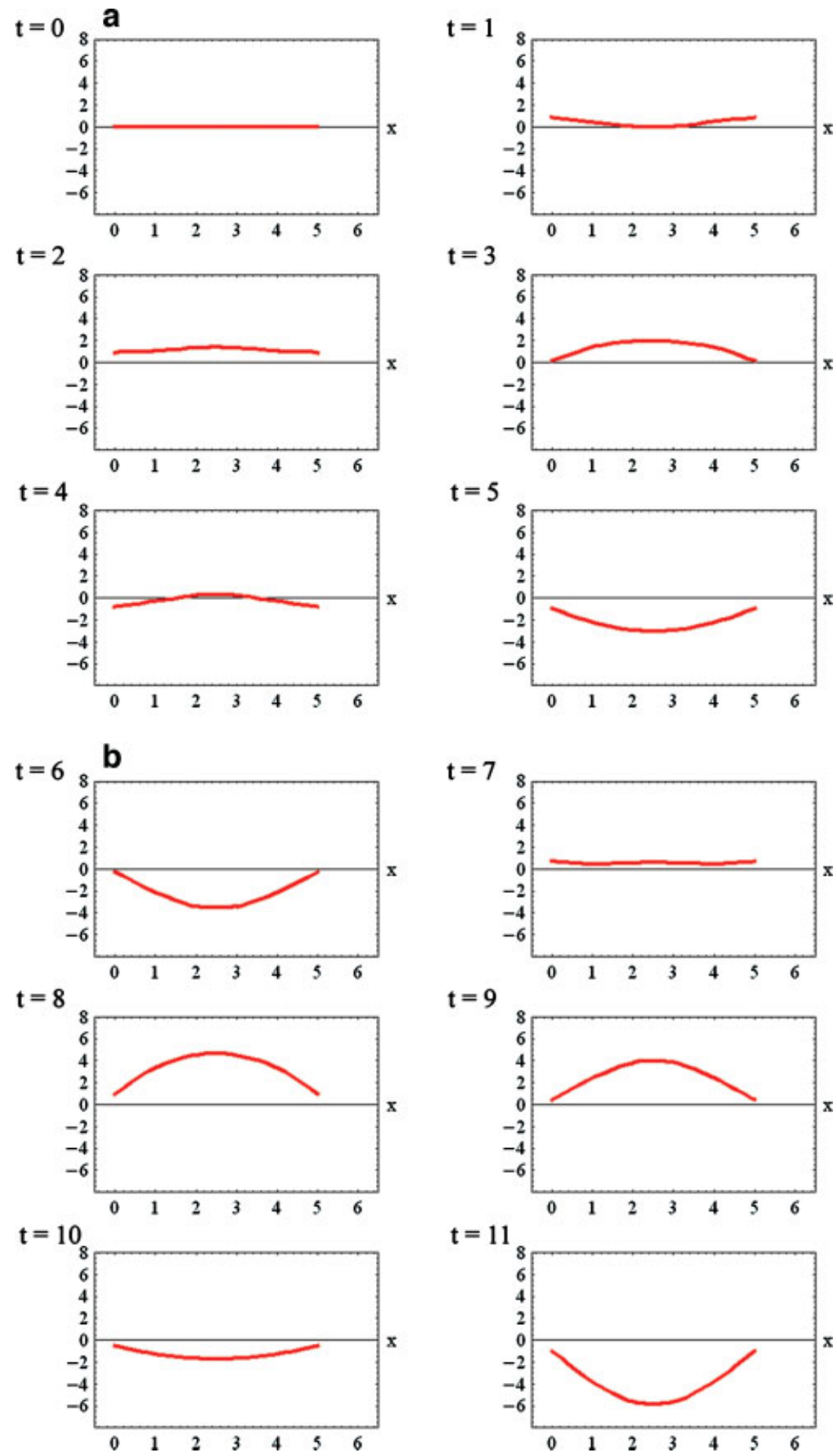

$t=3$

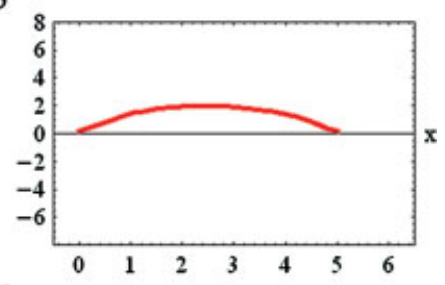

$t=5$
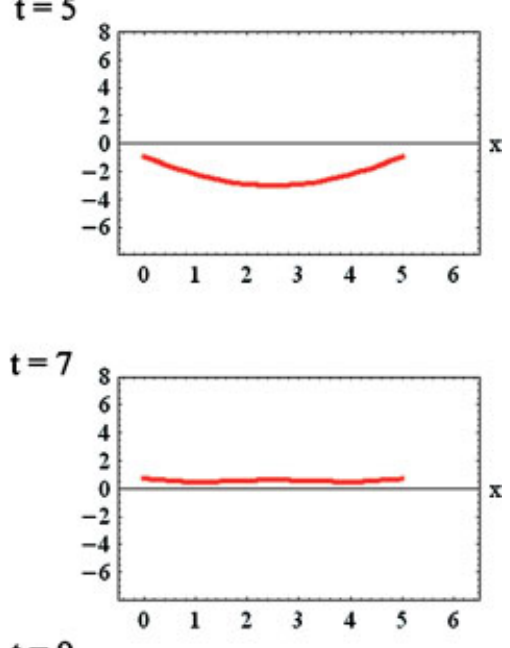

$t=9$
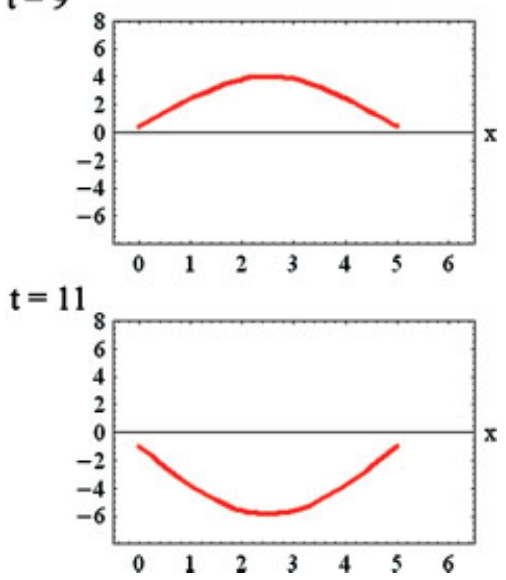

Figure 14 (a) In-phase response for a string subjected to support motions $(\mathrm{t}=0-5)$. (b) In-phase response for a string subjected to support motions $(\mathrm{t}=6-11)$. 

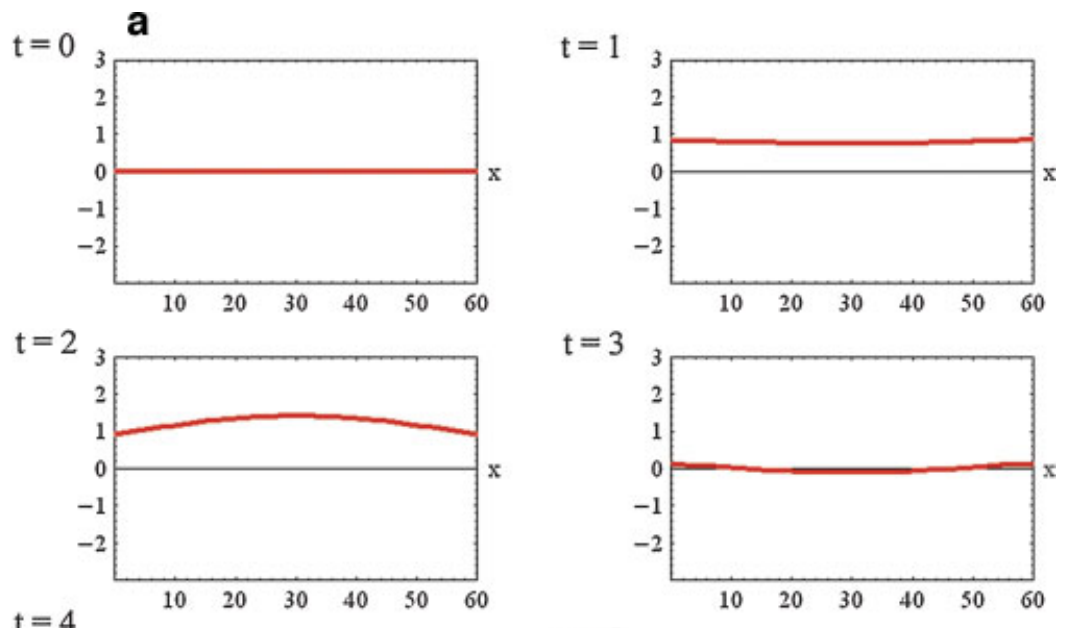

$\mathrm{t}=3$
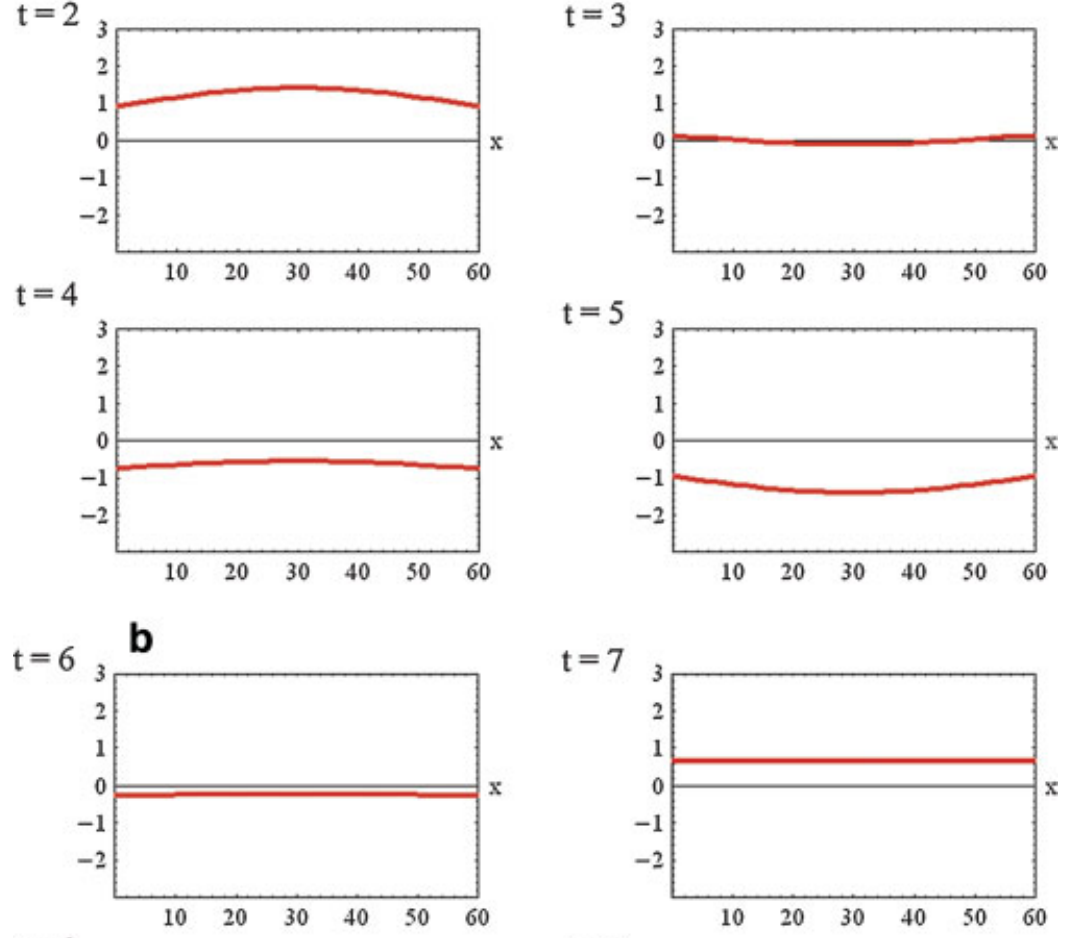

$\mathrm{t}=7$
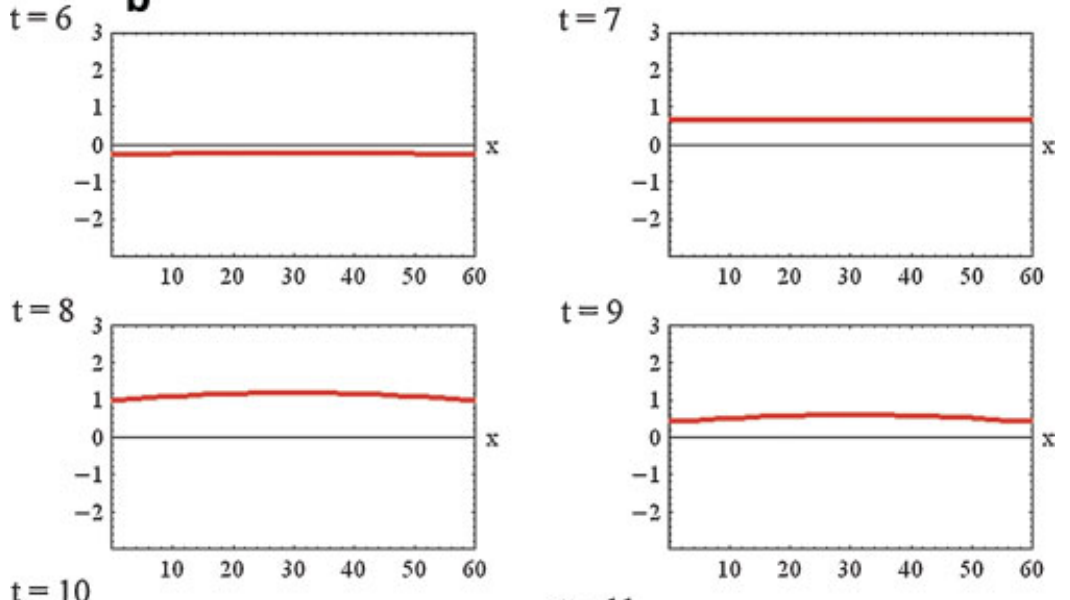

$\mathrm{t}=9$

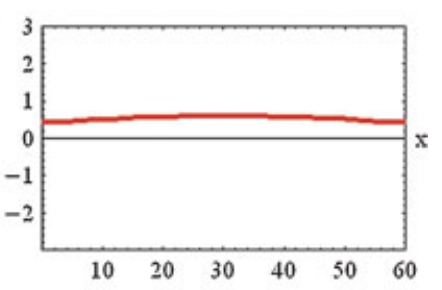

$\mathrm{t}=10$
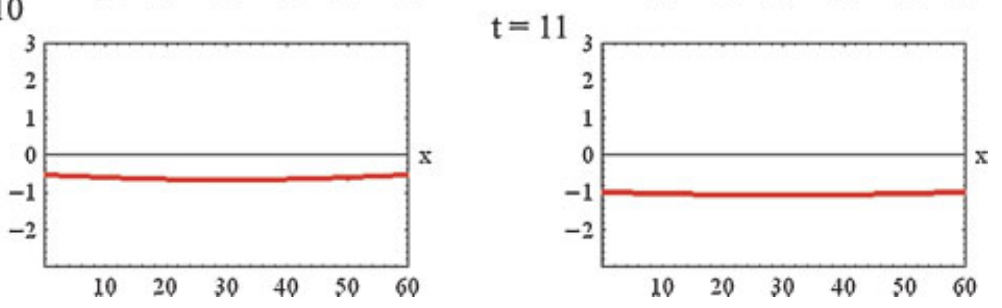

Figure 15 (a) In-phase response for a beam subjected to support motions $(t=0-5)$. (b) In-phase response for a beam subjected to support motions $(t=6-11)$. 
According to Equation (35) and BCs, the quasi-static [3] part solution can be obtained as shown below:

$$
U(x, t)=\sin (t)
$$

According to Equation (36), the total solution can be obtained as

$$
\begin{gathered}
u(x, t)=\sum_{n=1}^{\infty} \frac{10\left[\sin (t)+\left((-1)^{n}-1\right) \sin (n \pi t / 5)\right]}{n^{2} \pi^{2}} \\
\sin \left(\frac{n \pi x}{5}\right)+U(x, t)
\end{gathered}
$$

The animation can be produced by using the Mathematica, which is shown in Figure 14a and b.

\section{Example 6: A Beam Subjected to Support Motions}

The sixth example is a beam subjected to support motions. The initial conditions and the $\mathrm{BCs}$ of the support motion are the same with Equations (73) and (74), respectively. The input data are given as

$$
\begin{gathered}
c=1 \mathrm{~m} / \mathrm{s}, l=60 \mathrm{~m}, E I=2.45 \times 10^{9} \mathrm{Nm}^{2} \\
\rho A=2,400 \mathrm{~kg} / \mathrm{m}, \alpha=0.1381, \beta=0
\end{gathered}
$$

According to Equation (42) and the $\mathrm{BC}$, the quasistatic part [3] solution can be obtained as the same with Equation (75). In order to match the eigensystem, the $n$th natural mode $X_{n}(x)$ is expressed as

$$
X_{n}(x)=\sin \left(\frac{n \pi x}{l}\right)=\sin \left(\frac{n \pi x}{60}\right), \quad n=1,2, \ldots
$$

and the $n$th natural frequencies are

$$
\omega_{n}=\left(\frac{n \pi}{l}\right)^{2} \sqrt{\frac{E I}{\rho A}}=0.28066(n \pi)^{2}, \quad n=1,2, \ldots
$$

According to Equations (46) and (47), we can obtain a second order ODE

$$
\ddot{T}_{n}(t)+2 \xi_{n} \omega_{n} \dot{T}_{n}(t)+\omega_{n}^{2} q_{n}(t)=\frac{\ddot{F}_{n}(t)}{N}+\frac{2 \alpha \dot{F}_{n}(t)}{N}
$$

Then, we solve the second order ODE to obtain the function $T_{n}(t)$, which is shown below:

$$
\begin{aligned}
T_{n}(t)= & \frac{F_{n}(t)}{72,000}-\frac{1}{72,000 \omega_{n}^{d}} \int_{0}^{t} \mathrm{e}^{-\xi_{n} \omega_{n}(t-\tau)} \\
& \sin \left[\omega_{n}^{d}(t-\tau)\right]\left[\omega_{n}^{2} F_{n}(\tau)\right] \mathrm{d} \tau
\end{aligned}
$$

where

$$
\begin{gathered}
F_{n}(t)=\frac{144,000}{n \pi}\left[(-1)^{n} \sin (t)-\sin (t)\right] \\
\omega_{n}^{d}=\omega_{n} \sqrt{1-\left(\frac{0.0498562}{n^{2}}\right)^{2}}
\end{gathered}
$$

Therefore, the animation of solution of a beam subject to support motions is shown in Figure 15a and b. All Mathematica programs can be obtain in http:// ind.ntou.edu.tw/ msvlab/.

\section{CONCLUSIONS}

In this article, we have solved the one-dimensional wave problems by using the D'Alembert solution in conjunction with the diamond rule for infinite and semi-infinite strings with mass, damper, and spring. For the finite-string problems, three approaches, the diamond rule, the image method and the series solution, were employed to derive the analytical solutions. Besides, support motion problems for the string and beam were also solved by using the quasistatic decomposition method. All the results were shown in animation by using the Mathematica software. This display may be useful for students and engineers to capture the wave phenomena. Based on the animation, students can easily capture the sensitivity of response due to each parameter, for example, $m, c, k, \xi, E I$, and impedance ratio. This article provides the animation by using the Mathematica software. This can help teachers in the classroom using the multi-media equipment. We can provide animations where dynamic phenomenon can be well captured by students.

\section{REFERENCES}

[1] Beltzer, A. I. Engineering analysis via symbolic computation-a breakthrough, Appl Mech Rev 43 (1990), 119-127.

[2] Hard V. G. and Shi, J. Y. Effects of initial deformation in thin tubes on wave reflection and transmission at mechanical mismatch, J Sound Vib 188 (1995), 461-478.

[3] Chen J. T. and Jeng, Y. S. Dual series representation and its applications to a string subjected to support motions, Adv Eng Softw 27 (1996), 227-238.

[4] Chen, J. T. Hong, H. K. Yeh, C. S. and Chyuan, S. W. Integral representations and regularizations for a divergent series solution of a beam subjected to support motions, Earthquake Eng Struc 25 (1996), 909-925. 
[5] Ghidaui M. S. and Karney, B. W. Modified transformation and integration of $1 \mathrm{D}$ wave equations, J Hydraul Eng 121 (1995), 758-760.

[6] Wu C. M. and Lundberg, B. Reflection and transmission of the energy of harmonic elastic waves in a bent bar, J Sound Vib 190 (1996), 645659.

\section{BIOGRAPHIES}

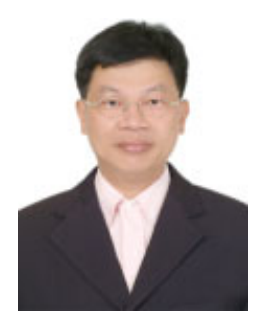

Jeng-Tzong Chen, born in 1962, received a BS degree in Civil Engineering, an MS in Applied Mechanics, and a $\mathrm{PhD}$ in Civil Engineering, respectively, in 1984, 1986, and 1994, from National Taiwan University, Taipei, Taiwan, ROC. He had worked as a research assistant in the Structural Division of the Department of Rocket and Missile System, Chung Shan Institute of Science and Technology, from 1986 to 1990. In 1994, he was invited to be an associate professor in the Department of Harbor and River Engineering, National Taiwan Ocean University, Keelung, Taiwan, R.O.C. He was promoted to full professor in 1998. Later in 2004, he was selected to be the distinguished professor. Furthermore, he was selected as the lifetime distinguished professor in 2007. His major interest is computational mechanics. He had derived the theory of dual integral equations for boundary value problems with degenerate boundary. Prof. Chen also developed four dual BEM programs for the BVPs of Laplace equation, Helmholtz equation, bi-Helmholtz and modified Helmhotlz equation and Navier equation. Recently, he also employed the null field integral equations to solve BVPs with circular boundaries. He wrote two books in Chinese on dual BEM and FEM using MSC/NASTRAN, respectively. He was invited to give plenaray and keynote lecturestwice in World Congress on Computational Mechanics (WCCM4 in Buenos Aries and WCCM5 in Vienna) and FEM/BEM 2003 in St. Petersburg, Russia. Also, he is the editor of Journal of the Chinese Institute of Civil and Hydraulic Engineering. He has been the Editor of Journal of Marine Science and Technology and the guest editor of J. Chinese Institute of Engineers. Several times he has won Outstanding Research Awards from National Science Council,
[7] Lee, S. K. Mace, B. R. and Brennan, M. J. Wave propagation, reflection and transmission in non-uniform one-dimensional waveguides, J Sound Vib 304 (2007), $31-49$.

[8] Kaw A. and Ho, S. On introducing approximate solution methods in theory of elasticity, Comput Appl Eng Educ 14 (2006), 120-134.

Taiwan. He also won the Wu, Ta-You Memorial Award in 2002. He is currently an editorial board member of five international journals. Until now, he has published more than $125 \mathrm{SCI}$ articles on BEM and FEM in technical Journals. Near 587 citings can be found. Boundary element method is one focus of Professor Chen's research interests. Others may be categorized into three areas. One is vibration and acoustics, another is computational mechanics and the other is engineering and mathematical education. E-mail: jtchen@mail. ntou.edu.tw, URL: http://ind.ntou.edu.tw/ msvlab

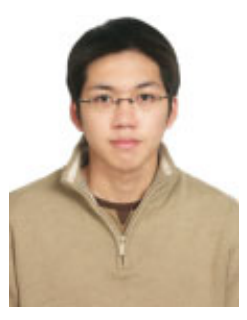

Ke-Shun Chou was born in Taiwan on Jan. 12,1983 . He is currently a master's degree student and a member of the Mechanics, Sound and Vibration Laboratory at the Department of Harbor and River Engineering at National Taiwan Ocean University. His research focuses on the application of the null-field BIEM. E-mail: m95520083@mail. ntou.edu.tw

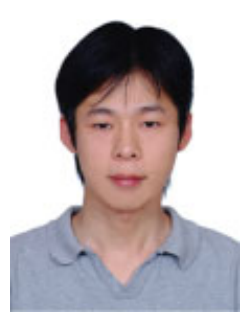

Shing-Kai Kao, born in 1978, is currently a master's degree student and a member of the Mechanics, Sound and Vibration Laboratory at the Department of Harbor and River Engineering at National Taiwan Ocean University. His interest is on the applications of Hilbert transform and $\mathrm{K}-\mathrm{K}$ transform to engineering problems. E-mail: m96520009@mail.ntou.edu.tw 